\title{
Development and validation of a claims- based measure as an indicator for disease status in patients with multiple sclerosis treated with disease-modifying drugs
}

Michael Munsell', Molly Frean ${ }^{1}$, Joseph Menzin ${ }^{1 *}$ and Amy L. Phillips ${ }^{2}$

\begin{abstract}
Background: Administrative healthcare claims data provide a mechanism for assessing and monitoring multiple sclerosis (MS) disease status across large, clinically representative "real-world" populations. The estimation of MS disease status using administrative claims can be a challenge, however, due to a lack of detailed clinical information. Retrospective claims analyses in MS have traditionally used rates of MS relapses to approximate disease status. Healthcare costs may be alternate, broader claims-based indicators of disease activity because costs reflect multiple facets of care of patients with MS, and there is a strong correlation between quality of life of patients with MS and costs of the disease. This study developed, tested, and validated a healthcare cost-based measure to serve as an indicator of overall disease status in patients with MS treated with disease-modifying drugs (DMDs) utilizing administrative claims.
\end{abstract}

Methods: Using IMS Health Real World Data Adjudicated Claims - US data (January 2006-June 2013), a negative binomial regression predicted annual all-cause medical costs. Coefficients reaching statistical significance $(p<0.05)$ and increasing costs by $\geq 5 \%$ were selected for inclusion into an MS-specific severity score (scale of 0 to 100). Components of the score included rehabilitation services, altered mental state, pain, disability, stiffness, balance disorder, urinary incontinence, numbness, malaise/fatigue, and infections. Coefficient weights represented each predictor's contribution. The predictive model was derived using 50\% of a random sample and tested/validated using the remaining 50\%.

Results: Average overall predicted annual total medical cost was $\$ 11,134$ (development sample, $n=11,384$, vs. $\$ 10,528$ actual) and $\$ 11,303$ (validation sample, $n=11,385$, vs. $\$ 10,620$ actual). The model had consistent bias (approximately $+\$ 600$ or $+6 \%$ of actual costs) for both samples. In the validation sample, mean MS disease status scores were 0.24 , 8.95, and 21.77 for low, medium, and high tertiles, respectively. Mean costs were most accurately predicted among less severe patients ( $\$ 5243$ predicted vs. $\$ 5233$ actual cost for lowest tertile).

Conclusion: The algorithm developed in this study provides an initial step to helping understand and potentially predict cost changes for a commercially insured MS population.

Keywords: Multiple sclerosis, Disease status measure, Retrospective database, Validation, Costs

\footnotetext{
*Correspondence: jmenzin@bhei.com

'Boston Health Economics, Inc., 20 Fox Road, Waltham, MA 02451, USA

Full list of author information is available at the end of the article
} International License (http://creativecommons.org/licenses/by/4.0/), which permits unrestricted use, distribution, and reproduction in any medium, provided you give appropriate credit to the original author(s) and the source, provide a link to the Creative Commons license, and indicate if changes were made. The Creative Commons Public Domain Dedication waiver (http://creativecommons.org/publicdomain/zero/1.0/) applies to the data made available in this article, unless otherwise stated. 


\section{Background}

Multiple sclerosis (MS) is an inflammatory-mediated, chronic neurodegenerative disease characterized by a range of symptoms including fatigue, impaired motor skills, blurred vision, bladder and bowel dysfunction, and cognitive impairment [1, 2]. The disease has a highly variable prognosis causing early severe disabilities in some patients, but leaving others ambulatory and functional for many years [3, 4]. Comorbidities are also highly prevalent in the MS population, and comorbid disease is recognized as a critical issue in MS given the breadth of adverse impacts with which it is associated [5]. The identification of patients with varying levels of overall disease status is important to help select patient populations most likely to benefit from interventions and to assess the value and effectiveness of treatments [6].

Administrative healthcare claims data provide a mechanism for assessing and monitoring MS disease status in patients with MS across large, clinically representative "real-world" populations [7-9]. Retrospective claims analyses in MS have traditionally used the rates of MS relapses (defined as MS-related hospitalizations, emergency room [ER] visits, or outpatient visits with pharmacy claims for a corticosteroid) as proxy measures for MS disease status [10-19]. Relapses alone, however, do not appropriately capture changes in disease progression and impairment [20]. For instance, as patients with MS progress over time, the number of relapses appears to decrease, despite worsening health status [20]. The estimation of MS disease status using administrative claims can be a challenge, however, due to a lack of detailed clinical information. Retrospective claims analyses in MS have traditionally used rates of MS relapses to approximate disease status. Furthermore, traditional medical models of impairment and disability in MS provide only an incomplete summary because they omit the consideration of comorbidities, secondary conditions, and health behaviors, which may influence the quality of life and disease burden of patients with MS along with biologic variables [21, 22].

Indicators of disease status that incorporate multiple facets of MS may permit assessment of the health status of the patient at a wider level [13, 23-26]. Healthcare costs may be alternate, broader claimsbased indicators of disease activity because costs reflect multiple facets of care of patients with MS, and there is a strong correlation between quality of life of patients with MS and costs of the disease [27, 28]. This study utilized an administrative claims dataset to develop, test, and validate a healthcare costs-based measure to serve as an indicator of disease status in patients with MS treated with disease-modifying drugs (DMDs).

\section{Methods}

\section{Data source and patient population}

This retrospective database study used IMS Health Real World Data (RWD) Adjudicated Claims - US data from January 1, 2006 to June 30, 2013. The IMS Health RWD Adjudicated Claims - US database includes complete, adjudicated insurance data, including complete inventory of a patient's prescriptions, inpatient hospital, and outpatient medical claims. The database consists primarily of patients with commercial health insurance and can thus under-represent the patients with government-paid health insurance (Medicaid or Medicare) relative to patients with private commercial insurance. The database includes $\sim 150$ million patients with a medical benefit, and a subset of 95 million patients with both medical and pharmacy benefits.

Patients were aged 18-64 years, had at least one MS diagnosis claim (International Classification of Diseases, Ninth Revision, Clinical Modification [ICD-9-CM] code: 340.xx), and at least one claim for a DMD between January 1, 2007 and June 30, 2012. The date of the first DMD prescription was designated as the index date. Patients were included if they had continuous eligibility 12 months pre- and post-index. Patients were excluded if they had any indication of pregnancy.

\section{Model development}

The data were divided into two samples: an original development sample and a validation/test sample (each comprised $50 \%$ of the total patient population). Patients were randomized using the "surveyselect" procedure in Statistical Analysis Software (SAS). A "seed" was set for the randomization process; therefore, the same patients were assigned to the same group every time the analysis was run (i.e., results were therefore replicable). The goal was to create claims-based measures of disease status, one specific to MS and the other focused on general health, using various comorbidity and MS symptomrelated codes, as well as additional variables (i.e., sex, age, census region, adherence, newly treated). The general health measure, which uses common Clinical Classification System (CCS) and Charlson criteria, is well-suited for the population as it provides a more complete view of the MS patient's health, and general health concerns can greatly increase costs among the MS population. MS and a general health measures were included in order to ensure the range of inputs that could affect the algorithm were captured. Costs were used as a proxy for disease status. The analysis evaluated healthcare costs using constant US dollars (i.e., costs were adjusted for inflation using the medical care component of the Consumer Price Index).

Multiple steps were implemented to achieve this goal. First, a negative binomial regression analysis was 
performed to predict all-cause total direct medical costs (excluding DMD costs) during the follow-up period. Regression covariates included 16 MS-related condition indicators (identified by diagnosis codes), 18 CCS codes, and 17 Charlson-Deyo comorbidities. Condition coefficients that reached both statistical $(p \leq 0.05)$ and economic (MS condition indicators, $\geq 5 \%$ increase in costs; general condition indicators [CCS and Charlson-Deyo], $\geq 20 \%$ increase in costs) significance were included in two normalized scores: an MS score and a general health score (Table 1). Additional file 1: Table S1 and Additional file 2: Table S2 provide the regression results and details of the MS and general score composition that were obtained in the development of the measures.

Secondly, coefficients included in each score were reweighted on a scale from 0 to 100 , such that weights represented each predictor's relative contribution to disease status, as measured by costs (Table 1). Finally, the original negative binomial regression model was reevaluated to predict costs as a function of the two scores, together with remaining covariates not included in the models for the MS or general health score. The fit of the two models was compared using Bayesian information criteria (BIC). The analysis demonstrated that the model including the MS and general health score was superior to the full regression model at predicting total direct medical costs (score model BIC: 227,340, full model: 227,508; a BIC difference $>10$ demonstrates very strong evidence) [29].

\section{Model validation and testing}

As the scores are intended to represent disease status as measured by costs, the MS score, the general health score, and the model combining them were tested and validated in the remaining $50 \%$ of the patient population

Table 1 MS and general health score components and points

\begin{tabular}{|c|c|c|c|}
\hline \multicolumn{2}{|l|}{ MS score } & \multicolumn{2}{|l|}{ General health score } \\
\hline Parameter & Points & Parameter & Points \\
\hline Rehabilitation & 25.597 & Myocardial infarction (Charlson) & 20.823 \\
\hline Altered mental state & 15.802 & Metastatic solid tumor (Charlson) & 19.027 \\
\hline Pain & 12.946 & Any primary malignancy (Charlson) & 12.172 \\
\hline Disability & 10.000 & Drug/device complication (CCS) & 10.453 \\
\hline Balance disorder & 9.211 & $\begin{array}{l}\text { Diabetes with chronic } \\
\text { complications (Charlson) }\end{array}$ & 8.937 \\
\hline Stiffness & 8.651 & Hematologic (CCS) & 6.251 \\
\hline Urinary incontinence & 6.366 & Gastrointestinal disease (CCS) & 5.891 \\
\hline Numbness & 4.779 & Psychiatric (CCS) & 5.785 \\
\hline Malaise and fatigue & 4.271 & Rheumatologic (Charlson) & 5.424 \\
\hline Infections & 2.377 & Genitourinary (CCS) & 5.238 \\
\hline
\end{tabular}

CCS Clinical Classification System, Charlson Charlson-Deyo comorbidities, MS multiple sclerosis (validation/test population) by assessing the relationship between the two scores and both the predicted and actual costs.

Patients were divided into separate MS and general health score tertiles (i.e., low, medium, and high disease status based on MS or general health score), and predicted and actual costs were summarized for each tertile. Tertiles were generated by ranking all patients according to their disease status score and then dividing the total population into three equal groups, with group cut-offs defined by score ranking. Patients with tied values were grouped into the same tertile. Tertiles were selected for ease of interpretation and because they effectively presented changes in MS/general disease status scores, as the distribution of patients across tertiles was relatively even. An increase in the proportion of patients with a given condition could be seen in each tertile.

All-cause total direct healthcare cost measures were summarized for each tertile using mean, standard deviation (SD), median, interquartile range, and minimum/ maximum. Statistical testing was employed to test the significance of difference in predicted versus actual costs between score groups. Differences between actual and predicted costs were assessed using Wilcoxon-MannWhitney tests for both the MS and general health score tertiles. Separate analyses were completed for both MS and general health score tertiles.

Additional validity testing was employed using general measures of model error. The bias and absolute prediction error of the model were calculated for both the original model sample and the remaining 50\% validation sample. This exercise was conducted to ensure that model error was consistent when using different patient populations. The equations below were used in the bias mean absolute prediction error (MAPE) analyses. These types of prediction accuracy measures have been used in previous health economic studies (e.g., Austin 2003 compared the accuracy of different regression models used to predict coronary artery bypass graft surgery medical costs) [30].

$$
\begin{aligned}
& \text { Bias }=(\text { mean of individual predictions })-(\text { mean of actual observations }) . \\
&=\frac{1}{n} \sum_{k} \hat{Y}_{k}-\frac{1}{n} \sum_{k} Y_{k} \\
& \text { MAPE }=\frac{1}{n} \sum_{k}\left|\hat{Y}_{k}-Y_{k}\right|
\end{aligned}
$$

\section{Exploration of MS/general health score composition}

The proportion of patients with each condition used to calculate the MS and general health score was evaluated for each MS/general health score tertile. This analysis was conducted to determine how disease status factors change as MS/general health score increases. 


\section{Results}

A total of 11,384 patients (50\%) were included in the original development population, and 11,385 patients (50\%) were included in the validation/test population.

A breakdown of the number of patients included in each MS and general health score tertile, as well as overall MS/general health scores for each tertile, are shown in Tables 2 and 3.

Mean MS scores were 0.24 , 8.95, and 21.77 in the low, medium, and high MS disease status tertiles, respectively. Patients in the low tertile had a median score of 0 and maximum score of 2.38, corresponding to the presence of an infection. Patients who experienced the three condition indicators associated with the highest scores rehabilitation services, altered mental state, and pain were all categorized in the highest tertile. Mean general health scores were $0.00,5.72$, and 16.07 for the low, medium, and high tertiles, respectively. No patients in the lowest tertile had any general health score condition indicators, and patients with any of the five condition indicators associated with the greatest score were all grouped in the highest tertile. Average annual predicted and actual costs in the overall population (i.e., not stratified by disease status tertile) for each group are shown in Fig. 1.

Bias was similar in both models, with predicted costs being approximately $6 \%$ higher than actual costs. The mean (SD) absolute prediction error was consistent across both populations: $\$ 7274(15,306)$ in the original development population and $\$ 7387(17,670)$ in the validation/test population.

Average annual predicted and actual costs within the validation/test population for the MS score and the general health score disease status tertiles are shown in Table 4.

The model predicted average costs most accurately for patients in the lowest disease status tertiles (bias of 0.21.9\%; Fig. 2).

A bias of $11.1-12.4 \%$ ( $\$ 2567$ for MS score, $\$ 2418$ for general health score) was recorded in the high disease status tertiles (Fig. 2). Differences in predicted vs. actual costs were significantly different across disease status tertiles for both MS and general health scores $(p<0.0001$ for all).

\section{Discussion}

Overall (i.e., without stratification by disease status tertiles), the average predicted annual direct healthcare cost was $\$ 11,134$ for the original development sample (vs. $\$ 10,528$ actual cost) and $\$ 11,303$ for the validation/ test sample (vs. $\$ 10,620$ actual cost). Therefore, the model had consistent bias (approximately $\$ 600$, or $6 \%$ of actual costs) for both samples. In Austin 2003, other regression-based prediction models had similar degrees of bias [30].

The mean absolute prediction error also remained consistent in both populations (approximately $\$ 7000$ for

Table 2 MS score and MS score component frequency within the validation/test population

\begin{tabular}{|c|c|c|c|}
\hline \multirow[b]{3}{*}{ Patients, $n$} & \multicolumn{3}{|c|}{ MS disease status group } \\
\hline & Low tertile & Medium tertile & High tertile \\
\hline & 3902 & 3801 & 3682 \\
\hline \multicolumn{4}{|l|}{ MS score } \\
\hline Mean (SD) & $0.24(0.71)$ & $8.95(2.46)$ & $21.77(9.23)$ \\
\hline Median (IQR) & $0.00(0.00-0.00)$ & $10.00(9.05-10.00)$ & $19.21(14.78-23.99)$ \\
\hline Minimum & 0 & 4.27 & 12.92 \\
\hline Maximum & 2.38 & 12.38 & 91.35 \\
\hline \multicolumn{4}{|c|}{ MS score components, $n$ (\%) } \\
\hline Rehabilitation & 0 & 0 & $135(3.7)$ \\
\hline Altered mental state & 0 & 0 & $133(3.6)$ \\
\hline Pain & 0 & 0 & $370(10.0)$ \\
\hline Disability & 0 & $2409(63.4)$ & $3369(91.5)$ \\
\hline Stiffness & 0 & $4(0.1)$ & $115(3.1)$ \\
\hline Balance disorder & 0 & $362(9.5)$ & $1823(49.5)$ \\
\hline Urinary incontinence & 0 & $126(3.3)$ & $425(11.5)$ \\
\hline Numbness & 0 & $317(8.3)$ & $1223(33.2)$ \\
\hline Malaise and fatigue & 0 & $686(18.0)$ & 1892 (51.4) \\
\hline Infections & $388(9.9)$ & $550(14.5)$ & 731 (19.9) \\
\hline
\end{tabular}


Table 3 General health score and general health score component frequency within the validation/test population

\begin{tabular}{|c|c|c|c|}
\hline \multirow[b]{3}{*}{ Patients, $n$} & \multicolumn{3}{|c|}{ General disease status group } \\
\hline & Low tertile & Medium tertile & High tertile \\
\hline & 4400 & 3426 & 3559 \\
\hline \multicolumn{4}{|l|}{ General health score } \\
\hline Mean (SD) & $0.00(0.00)$ & $5.72(0.31)$ & $16.07(7.22)$ \\
\hline Median (IQR) & $0.00(0.00-0.00)$ & $5.78(5.42-5.89)$ & $12.14(11.13-17.46)$ \\
\hline Minimum & 0 & 5.24 & 8.94 \\
\hline Maximum & 0 & 6.25 & 64.82 \\
\hline \multicolumn{4}{|c|}{ General health score components, $n(\%)$} \\
\hline Myocardial infarction & 0 & 0 & $47(1.3)$ \\
\hline Metastatic solid tumor & 0 & 0 & $45(1.3)$ \\
\hline Any primary malignancy & 0 & 0 & $363(10.2)$ \\
\hline Drug/device complication & 0 & 0 & $658(18.5)$ \\
\hline $\begin{array}{l}\text { Diabetes with chronic } \\
\text { complications }\end{array}$ & 0 & 0 & $159(4.5)$ \\
\hline Hematologic & 0 & $329(9.6)$ & $1038(29.2)$ \\
\hline Gastrointestinal disease & 0 & $1033(30.2)$ & $2301(64.7)$ \\
\hline Psychiatric & 0 & $1190(34.7)$ & $2090(58.7)$ \\
\hline Rheumatologic & 0 & $31(0.9)$ & $125(3.5)$ \\
\hline Genitourinary & 0 & 843 (24.6) & 1876 (52.7) \\
\hline
\end{tabular}

IQR interquartile range, MS multiple sclerosis, SD standard deviation

the original development and validation/test samples), demonstrating further validity of the model.

The mean absolute prediction error represents the average deviation of each individual predicted value from the actual value, and is therefore more sensitive to outliers than the measure of bias. However, the magnitude of the absolute prediction error in the analysis is comparable with the predictive cost models from Austin 2003, which resulted in a mean absolute error of approximately $\$ 6600$ for a dataset with mean actual costs of $\$ 17,900$ [30].
The slightly higher absolute prediction error in this study is most likely due to the magnitude of outliers from evaluating all-cause total direct medical costs vs. event-specific costs (i.e., maximum actual total cost in the analysis was $\$ 1,046,113$, while maximum actual surgery-specific cost was $\$ 166,461$ in Austin 2003 [30]).

On average, the model predicted costs most accurately among patients with lower disease status. Specifically, the model under-predicted costs by an average of $\$ 96$ for the low MS disease status tertile (mean predicted

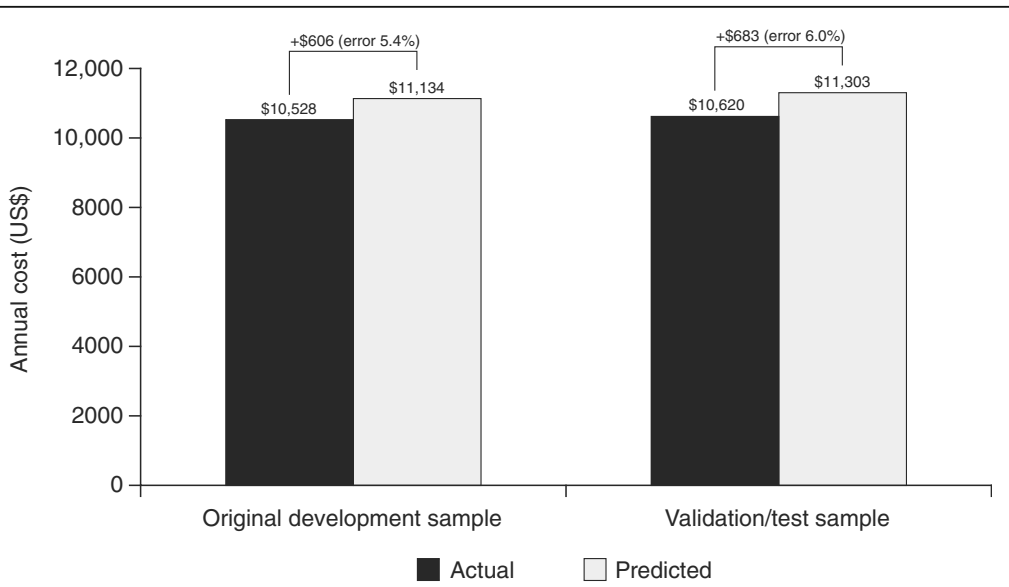

Fig. 1 Mean predicted vs. actual annual all-cause total medical costs for original development and validation/test samples* ${ }^{*}$ All costs adjusted to 2015 US dollars using the medical care component of the Consumer Price Index 
Table 4 Predicted vs. actual costs in the validation/test population, by MS or general health score disease status tertiles ${ }^{\mathrm{a}}$

\begin{tabular}{|c|c|c|c|}
\hline & \multicolumn{3}{|c|}{$\underline{\text { Disease status tertile }}$} \\
\hline & Low & Medium & High \\
\hline \multicolumn{4}{|l|}{ MS score } \\
\hline$n$ & 3902 & 3801 & 3682 \\
\hline \multicolumn{4}{|l|}{ Predicted costs, \$ } \\
\hline Mean (SD) & $5047(2867)$ & $8583(5397)$ & $20,743(34,041)$ \\
\hline Median (IQR) & $4269(3393-5712)$ & $7193(5359-10,042)$ & $12,941(8926-21,055)$ \\
\hline Minimum & 2149 & 2415 & 3642 \\
\hline Maximum & 55,398 & 82,177 & $1,046,113$ \\
\hline \multicolumn{4}{|l|}{ Actual costs, \$ } \\
\hline Mean (SD) & 5143 (9204) & $8923(12,770)$ & $18,176(27,936)$ \\
\hline Median (IQR) & $2688(1065-6052)$ & $5344(2617-10,210)$ & $10,215(5212-19,976)$ \\
\hline Minimum & 0 & 0 & 0 \\
\hline Maximum & 241,470 & 293,690 & 499,099 \\
\hline \multicolumn{4}{|c|}{ General health, score } \\
\hline$n$ & 4400 & 3426 & 3559 \\
\hline \multicolumn{4}{|l|}{ Predicted costs, \$ } \\
\hline Mean (SD) & $5243(2630)$ & $8202(4413)$ & $21,781(34,500)$ \\
\hline Median (IQR) & $4507(3471-6260)$ & $7175(5239-10,019)$ & $13,973(9567-22,598)$ \\
\hline Minimum & 2149 & 2638 & 3552 \\
\hline Maximum & 39,259 & 73,002 & $1,046,113$ \\
\hline \multicolumn{4}{|l|}{ Actual costs, \$ } \\
\hline Mean (SD) & $5233(7947)$ & $8455(10,609)$ & $19,363(29,432)$ \\
\hline Median (IQR) & $2950(1187-6195)$ & $5510(2686-10,350)$ & $10,725(5429-21,577)$ \\
\hline Minimum & 0 & 0 & 0 \\
\hline Maximum & 133,669 & 135,713 & 499,099 \\
\hline
\end{tabular}

IQR interquartile range, $M S$ multiple sclerosis, $S D$ standard deviation

${ }^{\text {a All }}$ costs adjusted to 2015 US dollars using the medical care component of the Consumer Price Index

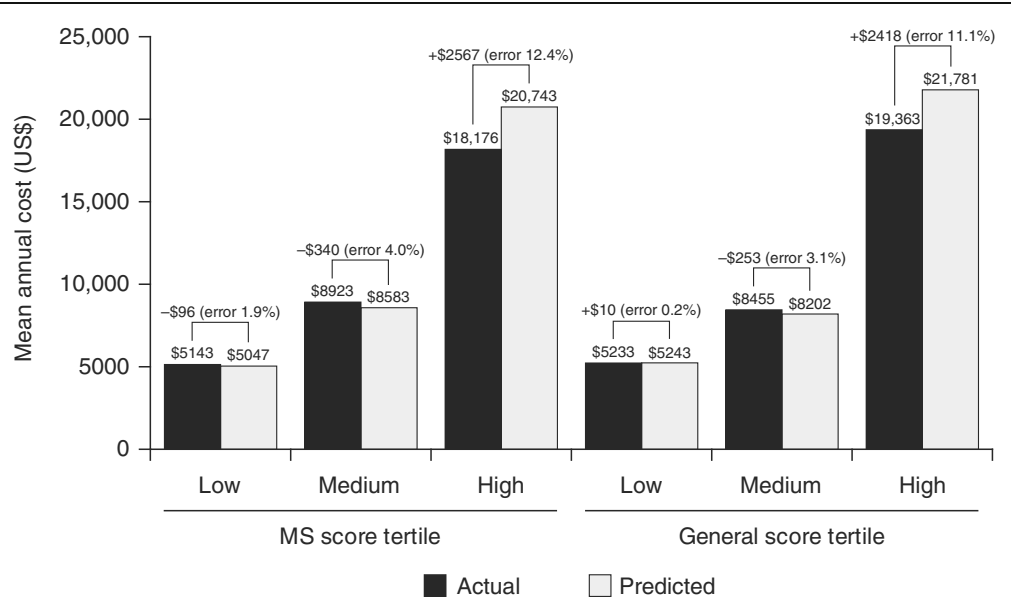

Fig. 2 Mean predicted vs. actual annual all-cause total medical costs and general health score tertile. MS multiple sclerosis*. ${ }^{*}$ All costs adjusted to 2015 US dollars using the medical care component of the Consumer Price Index 
$\$ 5047$; mean actual $\$ 5143$; bias $1.9 \%$ of actual) and overpredicted by only $\$ 10$ for the low general health score tertile (mean predicted \$5243; mean actual $\$ 5233$; bias $0.2 \%$ of actual). The difference between predicted and actual cost was approximately $\$ 2500$ for both the highest MS disease status and general health disease status tertiles (bias 11.1-12.4\% of actual). Differences in predicted vs. actual costs were significantly different $(p<0.0001)$ between disease status tertiles. The better prediction in the lowest tertile could be explained by the likely lower amounts of variation in this subpopulation compared with the other subpopulations.

The MS score model predicted annual all-cause total medical costs with acceptable estimations. While there does not appear to be a standard threshold for evaluating the accuracy of predictive cost models (i.e., most publications simply evaluate several different models and comment on relative accuracy by comparing model bias), the predictive model appears to be consistent with other published validated models. The bias of models used to predict coronary artery bypass graft surgery costs in the Austin 2003 publication ranged from $3.5 \%$ to $19.1 \%$ of the actual cost, with the negative binomial regression resulting in a $5.3 \%$ bias (vs. $6 \%$ bias in this analysis) [30]. An additional analysis that used a logistic regression to predict stroke treatment costs resulted in a 3\% bias (range 0-5\% of actual cost, depending on the subgroup analyzed), with the paper concluding that the predictive model's minimal bias directly confirmed its accuracy [31].

There are limitations in this analysis. The ICD-9-CM code for systemic MS does not distinguish between different MS types. Not all indicators of disease status may be captured in all-cause total medical costs as assessed by healthcare claims. Findings could be confounded by cost variations across settings, but relative relationships could be expected to hold. Also, a limited number of patients had very high MS or general health scores (mean disease status scores in the highest tertile did not exceed 25 out of a maximum score of 100 in either condition group). It is, therefore, difficult to evaluate the predictive accuracy of the model at the highest levels of MS disease status that were not present in the database. Finally, there is a possible lack of generalizability of the data given the inherent characteristics of claims databases and sample cohorts. The sample consisted of US patients with commercial claims. US patients with commercial claims are typically younger than 65 years of age, and most likely come from an employed population since they have commercial insurance; therefore, they may have better access to treatment. Also, the magnitude of claims is expected to be different in non-US patients; however, the conditions that are associated with high claims may still be relevant to other countries. Further research in other populations is warranted.

\section{Conclusions}

This analysis demonstrated that claims-based measures that incorporate MS-specific as well as general health components can be used as indicators for disease status in patients with MS treated with DMDs. The performance of the predictive model is consistent with other published validated models. Healthcare decision makers and researchers may use these models to better ascertain the disease status of patients with MS. This may help in identifying patients likely to benefit from intervention and help to assess the value and effectiveness of treatments.

\section{Additional files}

Additional file1: Table S1. Negative binomial regression predicting all-cause total costs (excluding DMD costs). Description of Data: Table S1 provides the regression results that were obtained in the development of the measures. (DOCX $15 \mathrm{~kb}$ )

Additional file 2: Table S2. MS and general score composition. Description of Data: Table S2 provides the details of the MS and general score composition that were obtained in the development of the measures. (DOCX $13 \mathrm{~kb}$ )

\section{Abbreviations}

BIC: Bayesian information criteria; CCS: Clinical Classification System; $D M D$ : Disease-modifying drug; ER: Emergency room; ICD-9CM: International Classification of Diseases, Ninth Revision, Clinical Modification; IQR: Interquartile range; MAPE: Mean absolute prediction error; MS: Multiple sclerosis.; SAS: Statistical Analysis Software.; SD: Standard deviation.

\section{Acknowledgements}

The authors thank Natalie Edwards of Health Services Consulting Corporation, Boxborough, MA, USA (supported by EMD Serono, Inc., Rockland, MA, USA [a business of Merck KGaA, Darmstadt, Germany]) for editorial assistance in drafting the manuscript, collating the comments of authors, and assembling tables and figures.

\section{Funding}

This study was supported by EMD Serono, Inc., Rockland, MA, USA (a business of Merck KGaA, Darmstadt, Germany) and Pfizer Inc., New York, NY, USA. EMD Serono, Inc., Rockland, MA, USA also funded editorial assistance in the preparation of this article.

\section{Availability of data and materials}

The data that support the findings of this study are available from IMS Health (Real World Data Adjudicated Claims - US data) but restrictions apply to the availability of these data, which were used under license for the current study, and so are not publicly available. Data are, however, available from the authors upon reasonable request and with permission of IMS Health.

\section{Authors' contributions}

$\mathrm{MM}, \mathrm{MF}$, and JM were involved in the design of the study, the analysis and interpretation of data, and in writing the manuscript. AP was involved in the design of the study, in the interpretation of data, and in writing the manuscript. All authors read and approved the final manuscript.

\section{Competing interests}

MM and JM are employees of Boston Health Economics, Inc., which received funding from EMD Serono, Inc., to conduct the analyses. MF is a former employee of Boston Health Economics, Inc. AP is an employee of EMD Serono, Inc., Rockland, MA, USA (a business of Merck KGaA, Darmstadt, Germany). The authors received no funding for their participation in the writing of the manuscript. 


\section{Consent for publication}

Not applicable.

\section{Ethics approval and consent to participate}

Ethics approval from an Institutional Review Board and Informed Consent were not required for this research database per US Department of Health and Human Services Exemption 4 (E4). The research involved the study of existing data and patients could not be identified directly or through identifiers linked to the subjects.

\section{Publisher's Note}

Springer Nature remains neutral with regard to jurisdictional claims in published maps and institutional affiliations.

\section{Author details}

'Boston Health Economics, Inc., 20 Fox Road, Waltham, MA 02451, USA. ${ }^{2}$ Health Economics \& Outcomes Research, EMD Serono, Inc., One Technology Place, Rockland, MA 02370, USA.

\section{Received: 4 November 2016 Accepted: 23 May 2017}

Published online: 05 June 2017

\section{References}

1. Compston A, Coles A. Multiple sclerosis. Lancet. 2002;359:1221-31.

2. National Multiple Sclerosis Society. Multiple Sclerosis FAQs. Last updated 2016 [cited 18 Mar 2016]. Available from: http://www.nationalmssociety.org/ What-is-MS/MS-FAQ-s

3. Baghizadeh S, Sahraian MA, Beladimoghadam N. Clinical and demographic factors affecting disease severity in patients with multiple sclerosis. Iran J Neurol. 2013;12:1-8.

4. Disanto G, Berlanga AJ, Handel AE, Para AE, Burrell AM, Fries A, et al. Heterogeneity in multiple sclerosis: scratching the surface of a complex disease. Autoimmune Dis. 2010;2011:932351

5. Marrie RA. Comorbidity in multiple sclerosis: some answers, more questions. Int J MS Care. 2016;18:271-2.

6. Signori A, Schiavetti I, Gallo F, Sormani MP. Subgroups of multiple sclerosis patients with larger treatment benefits: a meta-analysis of randomized trials. Eur J Neurol. 2015;22:960-6.

7. Chrischilles E, Schneider K, Wilwert J, Lessman G, O'Donnell B, Gryzlak B, et al. Beyond comorbidity: expanding the definition and measurement of complexity among older adults using administrative claims data. Med Care. 2014;52(Suppl 3):S75-84

8. Macaulay D, Sun SX, Sorg RA, Yan SY, De G, Wu EQ, et al. Development and validation of a claims-based prediction model for COPD severity. Respir med. 2013;107:1568-77.

9. Sung SF, Hsieh CY, Kao Yang YH, Lin HJ, Chen $\mathrm{CH}$, Chen YW, et al. Developing a stroke severity index based on administrative data was feasible using data mining techniques. J Clin Epidemiol. 2015;68: 1292-300.

10. Bergvall N, Makin C, Lahoz R, Agashivala N, Pradhan A, Capkun G, et al. Comparative effectiveness of fingolimod versus interferons or glatiramer acetate for relapse rates in multiple sclerosis: a retrospective US claims database analysis. Curr med res Opin. 2013;29:1647-56.

11. Bergvall N, Makin C, Lahoz R, Agashivala N, Pradhan A, Capkun G, et al. Relapse rates in patients with multiple sclerosis switching from interferon to fingolimod or glatiramer acetate: a US claims database study. PLoS One. 2014;9:e88472

12. Bergvall N, Lahoz R, Reynolds T, Korn JR. Healthcare resource use and relapses with fingolimod versus natalizumab for treating multiple sclerosis: a retrospective US claims database analysis. Curr med res Opin. 2014;30:1461-71.

13. Capkun G, Lahoz R, Verdun E, Song X, Chen W, Korn JR, et al. Expanding the use of administrative claims databases in conducting clinical real-world evidence studies in multiple sclerosis. Curr med res Opin. 2015;31:1029-39.

14. Castelli-Haley J, Oleen-Burkey MA, Lage MJ, Johnson K. Glatiramer acetate and interferon beta-1a for intramuscular administration: a study of outcomes among multiple sclerosis intent-to-treat and persistent-use cohorts. J med Econ. 2010;13:464-71.

15. Halpern R, Agarwal S, Dembek C, Borton L, Lopez-Bresnahan M. Comparison of adherence and persistence among multiple sclerosis patients treated with disease-modifying therapies: a retrospective administrative claims analysis. Patient Prefer Adherence. 2011;5:73-84.

16. Ivanova Jl, Bergman RE, Birnbaum HG, Phillips AL, Stewart M, Meletiche DM. Impact of medication adherence to disease-modifying drugs on severe relapse, and direct and indirect costs among employees with multiple sclerosis in the US. J med Econ. 2012;15:601-9.

17. Johnson BH, Bonafede MM, Watson C. Platform therapy compared with natalizumab for multiple sclerosis: relapse rates and time to relapse among propensity score-matched US patients. CNS Drugs. 2015;29:503-10.

18. Kozma CM, Phillips AL, Meletiche DM. Use of an early disease-modifying drug adherence measure to predict future adherence in patients with multiple sclerosis. J Manag Care Spec Pharm. 2014;20:800-7.

19. Tan H, Cai Q, Agarwal S, Stephenson JJ, Kamat S. Impact of adherence to disease-modifying therapies on clinical and economic outcomes among patients with multiple sclerosis. Adv Ther. 2011;28:51-61.

20. Goldman MD, Motl RW, Rudick RA. Possible clinical outcome measures for clinical trials in patients with multiple sclerosis. Ther adv Neurol Disord. 2010;3:229-39.

21. Marrie RA, Hanwell H. General health issues in multiple sclerosis: comorbidities, secondary conditions, and health behaviors. Continuum (Minneap Minn ). 2013;19:1046-57.

22. Mitchell AJ, Benito-Leon J, Gonzalez JM, Rivera-Navarro J. Quality of life and its assessment in multiple sclerosis: integrating physical and psychological components of wellbeing. Lancet Neurol. 2005;4:556-66.

23. Bueno AM, Sayao AL, Yousefi M, Devonshire $V$, Traboulsee A, Tremlett H. Health-related quality of life in patients with longstanding 'benign multiple sclerosis'. Mult Scler Relat Disord. 2015:4:31-8.

24. Kalincik T, Cutter G, Spelman T, Jokubaitis V, Havrdova E, Horakova D, et al. Defining reliable disability outcomes in multiple sclerosis. Brain. 2015;138: 3287-98.

25. Uitdehaag BM. Clinical outcome measures in multiple sclerosis. Handb Clin Neurol. 2014:122:393-404.

26. Zhang J, Waubant E, Cutter G, Wolinsky J, Leppert D. Composite end points to assess delay of disability progression by MS treatments. Mult Scler. 2014; 20:1494-501.

27. Henriksson F, Fredrikson S, Masterman T, Jonsson B. Costs, quality of life and disease severity in multiple sclerosis: a cross-sectional study in Sweden. Eur J Neurol. 2001:8:27-35.

28. Kobelt G, Berg J, Lindgren P, Fredrikson S, Jonsson B. Costs and quality of life of patients with multiple sclerosis in Europe. J Neurol Neurosurg Psychiatry. 2006;77:918-26.

29. Raftery AE. Bayesian model selection in social research. Sociol Methodol. 1995:25:111-63.

30. Austin PC, Ghali WA, Tu JV. A comparison of several regression models for analysing cost of CABG surgery. Stat med. 2003;22:2799-815.

31. Caro JJ, Huybrechts KF, Kelley HE. Predicting treatment costs after acute ischemic stroke on the basis of patient characteristics at presentation and early dysfunction. Stroke. 2001;32:100-6.

\section{Submit your next manuscript to BioMed Central and we will help you at every step:}

- We accept pre-submission inquiries

- Our selector tool helps you to find the most relevant journal

- We provide round the clock customer support

- Convenient online submission

- Thorough peer review

- Inclusion in PubMed and all major indexing services

- Maximum visibility for your research

Submit your manuscript at www.biomedcentral.com/submit
) Biomed Central 